\title{
Skew Semi-Heyting Algebras
}

\author{
Berhanu Assaye Alaba, Mihret Alamneh and Yeshiwas Mebrat
}

\begin{abstract}
In this paper, we introduce the concept of skew semi-Heyting algebra and extend the notions of semi-Heyting algebras. We characterize a skew semi-Heyting algebra as a skew Heyting algebra interms of a unique binary operation on which an induced binary operation is defined, and some algebraic properties on it. bra.

Index Terms-Skew semi-Heyting algebra, semi-Heyting alge-
\end{abstract}

\section{INTRODUCTION}

$\mathbf{T}$ HE foundation of modern theory of skew lattices can be found in Jonathan Leech's 1989 paper [1]. Leech [2], [3] showed that each right handed skew Boolean algebra can be embedded in to a generic skew Boolean algebra of partial functions from a given set to the co-domain $\{0,1\}$. Heyting algebra is a relatively pseudo-complemented distributive lattice which arises from non-classical logic, and it was first investigated by T. Skolem about 1920 [4]. A Heyting algebra named after a Dutch mathematician Arend Heyting was introduced by G. Birkhoff [5] and is developed by H. B. Curry about 1963. While Boolean algebras provide algebraic models of classical logic, Heyting algebras provide algebraic models of intuitionistic logic. The notion of skew Heyting algebra was introduced by Karin Cvetko-vah [6] as a generalization of Heyting algebra. In that paper, it is proved that a skew Heyting algebras form a variety and that the maximal lattice image of a skew Heyting algebra is a generalized Heyting algebra. H.P. Sankappanavar [7] in 2007 define and investigate a new (equational) class of algebras, which is called Semi-Heyting Algebras, as an abstraction from Heyting algebras. He showed that semi-Heyting algebras share with Heyting algebras some strong properties, like these algebras are: pseudocomplemented, distributive, congruences on them are determined by filters and every interval in a semiHeyting algebra is also pseudo complemented.

This paper consists of two sections. The first section describes preliminary concepts which can be used in proving lemmas, theorems and corollaries in the subsequent section. In the second section, we introduce the notion of skew semiHeyting algebra and present some basic arithmetical properties. In this section, we also characterize a skew semi-Heyting algebra as a skew Heyting algebra interms of a unique binary operation ${ }_{b} \rightarrow$ defined on $b \uparrow$ for any $b \in L$ such that ${ }_{b} \rightarrow$ is precisely the induced binary operation on $L$, and some algebraic properties of it.

Manuscript received June 9, 2017; accepted November 13, 2017

B. A. Alaba and M. Alamneh are with the Department of Mathematics, College of Science, Bahir Dar University, Bahir Dar, Ethiopia. Email: berhanu_assaye@yahoo.com

Y. Mebrat is with the Department of Mathematics, Faculty of Science, Debre Tabor University, Debre Tabor, Ethiopia. Email: yeshiwasmebrat@gmail. com

\section{PRELIMINARIES}

First, we give the necessary definitions and results on Heyting algebras, semi-Heyting algebras, skew lattices and skew Heyting algebras which will be used in the next section.

Definition 2.1 ([7]): An algebra $(L, \vee, \wedge, \rightarrow, 0,1)$ of type $(2,2,2,0,0)$ is called a Heyting algebra if it satisfies the following axioms:

(1) $(L, \vee, \wedge, 0,1)$ is a lattice with 0 and 1

(2) $x \wedge(x \rightarrow y)=x \wedge y$

(3) $x \wedge(y \rightarrow z)=x \wedge((x \wedge y) \rightarrow(x \wedge z))$

(4) $(x \wedge y) \rightarrow x=1$

for all $x, y, z \in L$.

Lemma 2.2 ([8]): Let $L$ be a Heyting algebra, then an equivalence relation $\theta$ on $L$ is a congruence relation if and only if for any $(a, b) \in \theta, d \in L$,

(1) $(a \wedge d, b \wedge d) \in \theta$

(2) $(a \vee d, b \vee d) \in \theta$

(3) $(a \rightarrow d, b \rightarrow d) \in \theta$

(4) $(d \rightarrow a, d \rightarrow b) \in \theta$.

Definition 2.3 ([7]): An algebra $(L, \vee, \wedge, \rightarrow, 0,1)$ is a semiHeyting algebra if the following conditions hold:

(SH1) $(L, \vee, \wedge, 0,1)$ is a lattice with 0 and 1

(SH2) $x \wedge(x \rightarrow y)=x \wedge y$

(SH3) $x \wedge(y \rightarrow z)=x \wedge((x \wedge y) \rightarrow(x \wedge z))$

(SH4) $x \rightarrow x=1$.

Theorem 2.4: [7] Let $L$ be a semi-Heyting algebra. Then for any $x, y, z \in L, L$ satisfies the following conditions:

(a) $x \wedge(y \rightarrow z)=x \wedge((x \wedge y) \rightarrow z)$

(b) $x \wedge(y \rightarrow z)=x \wedge(y \rightarrow(x \wedge z))$.

Definition 2.5 ([1]): A skew lattice is an algebra $\mathbf{L}=$ $(L ; \wedge, \vee)$ of type $(2,2)$ such that $\wedge$ and $\vee$ are both idempotent and associative, and they satisfy the following absorption laws: $x \wedge(x \vee y)=x=x \vee(x \wedge y)$ and $(x \wedge y) \vee y=y=(x \vee y) \wedge y$ for all $x, y \in L$.

The natural partial order can be defined on a skew lattice $\mathbf{L}$ by stating that $x \leq y$ if and only if $x \vee y=y=y \vee x$, or equivalently $x \wedge y=x=y \wedge x$ for $x, y \in L$. Also the natural preorder can be defined by $x \preceq y$ if and only if $y \vee x \vee y=$ $y$, or equivalently $x \wedge y \wedge x=x$ for $x, y \in L$ so that Green's equivalence relation $D$ is defined by $x D y$ if and only if $x \preceq y$ and $y \preceq x$ (see [7]).

Definition 2.6 ([6]): A skew lattice is called strongly distributive if for all $x, y, z \in L$ it satisfies the following identities: $x \wedge(y \vee z)=(x \wedge y) \vee(x \wedge z)$ and $(x \vee y) \wedge z=(x \wedge z) \vee(y \wedge z)$; and it is called co-strongly distributive if it satisfies the identities: $x \vee(y \wedge z)=(x \vee y) \wedge(x \vee z)$ and $(x \wedge y) \vee z=(x \vee z) \wedge(y \vee z)$.

Definition 2.7: [3], [6] A skew Lattice $L$ is called normal if $w \wedge x \wedge y \wedge z=w \wedge y \wedge x \wedge z$ and it is called conormal if $w \vee x \vee$ $y \vee z=w \vee y \vee x \vee z$ for all $w, x, y, z \in L$. 
A skew lattice which is both normal and conormal is called binormal skew lattice. If a skew lattice is strongly distributive, then it is normal. Dually if a skew lattice is costrongly distributive, then it is conormal (see [3], [6]).

Definition 2.8 ([6]): An algebra $\mathbf{L}=(L ; \vee, \wedge, \rightarrow, 1)$ of type $(2,2,2,0)$ is said to be a skew Heyting algebra whenever the following conditions are satisfied:

(1) $(L ; \vee, \wedge, 1)$ is a co-strongly distributive skew lattice with top 1.

(2) For any $a \in L$, an operation $\rightarrow_{a}$ can be defined on $a \uparrow=\{a \vee x \vee a \mid x \in L\}$ such that $\left(a \uparrow, \vee, \wedge, \rightarrow_{a}, 1, a\right)$ is a Heyting algebra with top 1 and bottom $a$.

(3) An induced binary operation $\rightarrow$ from $\rightarrow_{a}$ is defined on $L$ by

$$
x \rightarrow y=(y \vee x \vee y) \rightarrow_{y} y .
$$

Lemma 2.9 ([6]): Let $(L, \vee, \wedge, \rightarrow, 1)$ be a skew Heyting algebra and let $x, y, a \in L$ be such that $x, y \in a \uparrow$ hold. Then $x \rightarrow y=x \rightarrow_{a} y$.

\section{Skew Semi-Heyting Algebras}

In this section, we introduce the concept of skew semiHeyting algebra, characterize it as a skew Heyting algebra in terms of a unique binary operation on which an induced binary operation is defined and investigate some of its algebraic properties. Throughout this section, $L$ stands for a non empty set and we will use the following notations:

(i) For any $a \in L$ and a partial ordering $\leq$ on $L$, the set $\{x \in L \mid a \leq x \leq b\}$ is denoted by $[a, b]$,

(ii) If $L$ contains 0 for any $a \in L, \rightarrow_{a}$ is the binary operation defined on $[0, a]$,

(iii) For any $b, c \in L, b \rightarrow$ is the binary operation defined on $[b, c]$.

Definition 3.1: An algebra $(L ; \vee, \wedge, \rightarrow, 1)$ of type $(2,2,2,0)$ is said to be a skew semi-Heyting algebra whenever the following conditions are satisfied:

(1) $(L ; \vee, \wedge, 1)$ is a co-strongly distributive skew lattice with top 1.

(2) For any $a \in L$, an operation $a \rightarrow$ can be defined on $a \uparrow=$ $\{x \in L \mid a \leq x\}$ such that $(a \uparrow, \vee, \wedge, a \rightarrow, 1, a)$ is a semiHeyting algebra with top 1 and bottom $a$.

(3) An induced binary operation $\rightarrow$ from $a \rightarrow$ is defined on $L$ by

$$
x \rightarrow y=(y \vee x \vee y)_{y} \rightarrow y .
$$

Example 3.2: Let $L$ be a co-strongly distributive skew lattice and $b \in L$ such that $x, y \in b \uparrow$. Define a binary operation ${ }_{b} \rightarrow$ on $b \uparrow$ by

$$
x_{b} \rightarrow y= \begin{cases}1 & \text { if } x \leq y \\ y & \text { otherwise }\end{cases}
$$

One can show that $b \uparrow$ is a semi-Heyting algebra. Thus defining a binary operation $\rightarrow$ on $L$ induced from $b \rightarrow$ by, $x \rightarrow y=$ $(y \vee x \vee y)_{y} \rightarrow y$ makes $L$ a skew semi-Heyting algebra.

A semi-Heyting algebra is not necessarily a skew semiHeyting algebra. The following example justifies this.

Example 3.3: Let $L=\{0, x, 1\}$ be a chain with $0<x<1$ which is the lattice reduct of the two semi-Heyting algebras with the binary operation $\rightarrow$ defined on L by the tables given below. It can be routinely verified that the algebra $(L, \vee, \wedge, \rightarrow, 1)$ where the binary operation $\rightarrow$ defined by Table 1 is a skew semi-Heyting algebra. But $(L, \vee, \wedge, \rightarrow, 1)$ where the binary operation $\rightarrow$ defined by Table 2 is not a skew semiHeyting algebra, because when we apply the definition of $\rightarrow$ in Table 2 we obtain that $0 \rightarrow x=(x \vee 0 \vee x)_{x} \rightarrow x \Rightarrow 0 \rightarrow x=$ $x_{x} \rightarrow x \Rightarrow x=1$ which is impossible.

We now present some useful arithmetical properties of skew semi-Heyting algebras.

Theorem 3.4: Let $\mathrm{L}$ be a skew semi-Heyting algebra and $x, y, z \in L$. Then the following hold:
(a) $1 \rightarrow x=x$
(b) $x \rightarrow 1=1$
(c) $x \leq y \Rightarrow x \rightarrow y=1$
(d) $y \leq x \rightarrow y$
(e) $(x \vee y) \rightarrow x=y \rightarrow x$
(f) $x \leq y \leq z \Rightarrow y \leq x \rightarrow z$
(g) $(x \wedge y) \rightarrow x=1$
(h) $x \rightarrow(y \rightarrow x)=y \rightarrow(x \rightarrow y)$

Proof: (a) and (b) hold trivially.

(c) Suppose $x \leq y$. Then $x \rightarrow y=(y \vee x \vee y)_{y} \rightarrow y=1$

(d) $y \wedge(x \rightarrow y)=y \wedge\left((y \vee x \vee y)_{y} \rightarrow y\right)=y \wedge\{(y \wedge(y \vee x \vee$ $\left.y))_{y} \rightarrow y\right\}=y \wedge\left(y_{y} \rightarrow y\right)=y \wedge 1=y$. Moreover, since $y \vee x \vee$ $y$ and $y$ belongs to $y \uparrow,\left((y \vee x \vee y)_{y} \rightarrow y\right) \wedge y=y$

(e) $(x \vee y) \rightarrow x=(x \vee(x \vee y) \vee x)_{x} \rightarrow x=(x \vee y \vee x)_{x} \rightarrow x=y \rightarrow x$.

(f) Let $x \leq y \leq z$. Then $y \wedge(x \rightarrow z)=y \wedge\left((z \vee x \vee z)_{z} \rightarrow z\right)=$ $y \wedge z \wedge\left((z \vee x \vee z)_{z} \rightarrow z\right)=y \wedge z \wedge\left(z_{z} \rightarrow z\right)=y \wedge z=y$. Clearly, $(x \rightarrow z) \wedge y=y$

(g) $(x \wedge y) \rightarrow x=(x \vee(x \wedge y) \vee x)_{x} \rightarrow x=x_{x} \rightarrow x=1$.

(h) From (d) we have $x \leq y \rightarrow x$ and $y \leq x \rightarrow y$. Then by (c) we conclude that $x \rightarrow(y \rightarrow x)=1=y \rightarrow(x \rightarrow y)$.

Lemma 3.5: Let $L$ be a skew semi-Heyting algebra. Then for any $a \in L, a \uparrow$ is a distributive lattice.

Proof: Suppose L be a skew semi-Heyting algebra. Let $a \in L$. Then $a \uparrow$ is a semi-Heyting algebra. Hence $a \uparrow$ is a distributive lattice.

\begin{tabular}{|c|c|c|c|}
\hline$\rightarrow$ & 0 & $x$ & 1 \\
\hline 0 & 1 & 1 & 1 \\
\hline$x$ & 0 & 1 & 1 \\
\hline 1 & 0 & $x$ & 1 \\
\hline
\end{tabular}

TABLE I SKEW SEMI-HEYTING ALGEBRA

Lemma 3.6: Let $L$ be a skew semi-Heyting algebra and $x, y \in$ $L$. Then $x \vee y \in y \uparrow$ if and only if $y \vee x \in x \uparrow$. Whenever $x \vee y$ and $y \vee x$ both belongs to $y \uparrow$, then they are equal.

Proof: Suppose $x \vee y \in y \uparrow$. Since $y \in y \uparrow$, we have $y \vee(x \vee$ $y)=(x \vee y) \vee y$. Using associative property of skew lattice we obtain $y \vee x \vee y=x \vee y$. Thus $(y \vee x \vee y) \vee x=x \vee y \vee x$ so that $y \vee x=x \vee y \vee x$. Clearly $x \vee y \vee x \in x \uparrow$ for some $y \in L$ which implies that $y \vee x \in x \uparrow$. The converse holds analogously and the proof of the other condition is so simple.

Following the above lemma we have the next theorem. 
Theorem 3.7: Let $L$ be a skew semi-Heyting algebra and $x, y, z \in L$ such that $x \vee y \in y \uparrow$. Then the following hold.

(a) $x \rightarrow y=1 \Rightarrow x \vee y=y$ and $x \wedge y=x$

(b) $x \wedge(y \rightarrow z)=x \wedge((x \wedge y) \rightarrow z)$

(c) $x \wedge((x \rightarrow y) \rightarrow y)=x$

(d) $(x \rightarrow y) \wedge(y \rightarrow x)=1 \Rightarrow y \wedge x=y$

(e) $x \wedge(x \rightarrow y)=x \wedge y$

(f) $x \leq y \Rightarrow x \wedge(z \rightarrow y)=x \wedge y$

(g) $(x \vee y) \rightarrow(x \wedge y)=1 \Rightarrow x \wedge y=x$.

Proof: (a) Assume $x \rightarrow y=1$

$$
\begin{aligned}
& \Rightarrow \quad(y \vee x \vee y)_{y} \rightarrow y=1 \\
& \Rightarrow \quad(x \vee y) \wedge\left\{(y \vee(x \vee y))_{y} \rightarrow y\right\}=x \vee y \\
& \Rightarrow \quad(x \vee y) \wedge\left((x \vee y)_{y} \rightarrow y\right)=x \vee y \\
& \Rightarrow \quad x \vee y=y .
\end{aligned}
$$

Similarly we obtain that, $(y \vee x \vee y)_{y} \rightarrow y=1$

$$
\begin{aligned}
& \Rightarrow \quad x \wedge\left((y \vee x \vee y)_{y} \rightarrow y\right)=x \\
& \Rightarrow \quad x \wedge(x \vee y) \wedge\left((y \vee x \vee y)_{y} \rightarrow y\right)=x \\
& \Rightarrow \quad x \wedge(x \vee y) \wedge\left((x \vee y)_{y} \rightarrow y\right)=x \\
& \Rightarrow \quad x \wedge y=x .
\end{aligned}
$$

(b) $x \wedge(y \rightarrow z)$

$$
\begin{aligned}
= & x \wedge\left\{(z \vee y \vee z)_{z} \rightarrow z\right\} \\
= & x \wedge(x \vee z) \wedge\left\{(z \vee y \vee z)_{z} \rightarrow z\right\} \\
= & x \wedge(x \vee z) \wedge\left\{((x \vee z) \wedge(z \vee y \vee z))_{z} \rightarrow\right. \\
& ((x \vee z) \wedge z)\} \\
= & x \wedge\left\{\{((x \vee z) \wedge z) \vee((x \vee z) \wedge(y \vee z))\}_{z} \rightarrow z\right\} \\
= & x \wedge\left\{\{(z \vee((x \wedge y) \vee z))\}_{z} \rightarrow z\right\} \\
= & x \wedge((x \wedge y) \rightarrow z) .
\end{aligned}
$$

(c) $x \wedge((x \rightarrow y) \rightarrow y)$

$$
\begin{aligned}
& =x \wedge\left\{\left((y \vee x \vee y)_{y} \rightarrow y\right) \rightarrow y\right\} \\
& =x \wedge\left\{\left(y \vee\left((y \vee x \vee y)_{y} \rightarrow y\right) \vee y\right)_{y} \rightarrow y\right\} \\
& =\quad x \wedge(x \vee y) \wedge\left\{\left(y \vee\left((y \vee x \vee y)_{y} \rightarrow y\right) \vee y\right)_{y} \rightarrow y\right\} \\
& \left.=x \wedge\left\{y \vee\left\{(x \vee y) \wedge\left\{(x \vee y)_{y} \rightarrow y\right)\right\} \vee y\right\}_{y} \rightarrow y\right\} \\
& =x \wedge\left(y_{y} \rightarrow y\right) \\
& =x \wedge 1 \\
& =x .
\end{aligned}
$$

(d) $(x \rightarrow y) \wedge(y \rightarrow x)=1$

$$
\begin{aligned}
\Rightarrow & \left((y \vee x \vee y)_{y} \rightarrow y\right) \wedge\left((x \vee y \vee x)_{x} \rightarrow x\right)=1 \\
\Rightarrow & y \wedge\left\{\left((y \vee x \vee y)_{y} \rightarrow y\right) \wedge\left((x \vee y \vee x)_{x} \rightarrow x\right)\right\}=y \\
\Rightarrow & y \wedge\left((x \vee(y \vee x))_{x} \rightarrow x\right)=y \\
\Rightarrow \quad & y \wedge(y \vee x) \wedge\left((x \vee(y \vee x))_{x} \rightarrow x\right)=y \\
\Rightarrow \quad & y \wedge(y \vee x) \wedge\left\{\{(y \vee x) \wedge(x \vee(y \vee x))\}_{x} \rightarrow\right. \\
& (y \vee x) \wedge x)\}=y \\
\Rightarrow \quad & y \wedge\left((y \vee x)_{x} \rightarrow x\right)=y \\
\Rightarrow \quad & y \wedge(y \vee x) \wedge\left((y \vee x)_{x} \rightarrow x\right)=y \\
\Rightarrow & y \wedge x=y .
\end{aligned}
$$

(e) $x \wedge(x \rightarrow y)$

$$
\begin{aligned}
= & x \wedge\left((y \vee x \vee y)_{y} \rightarrow y\right) \\
= & x \wedge(x \vee y) \wedge\left\{((y \vee x) \vee(x \vee y))_{y} \rightarrow y\right\} \\
= & x \wedge(x \vee y) \wedge\left\{\{(x \vee y) \wedge((y \vee x) \vee(x \vee y))\}_{y} \rightarrow\right. \\
& ((x \vee y) \wedge y)\} \\
= & x \wedge(x \vee y) \wedge\left\{\{((y \vee x) \vee(x \vee y)) \wedge(x \vee y)\}_{y} \rightarrow y\right\} \\
= & x \wedge\left((x \vee y)_{y} \rightarrow y\right) \\
= & x \wedge y .
\end{aligned}
$$

(f) $x \wedge(z \rightarrow y)=x \wedge y \wedge\left((y \vee z \vee y)_{y} \rightarrow y\right)=x \wedge y$.

(g) Suppose $(x \vee y) \rightarrow(x \wedge y)=1$. Then $x \wedge((x \vee y) \rightarrow(x \wedge y))=$ $x$. From (b) we have $x \wedge((x \wedge(x \vee y)) \rightarrow(x \wedge y)=x$. Thus, applying (e) on $x \wedge(x \rightarrow(x \wedge y))=x$ yields $x \wedge(x \wedge y)=x$. Therefore $x \wedge y=x$.

Next we give a characterization of skew semi-Heyting algebra.

Theorem 3.8: Let $\mathrm{L}$ be a skew semi-Heyting algebra and $a \in L$ such that $x, y, z \in a \uparrow$. Then the following are equivalent:

(a) $L$ is a skew Heyting algebra

(b) $x \leq y \Rightarrow x_{a} \rightarrow y=1$

(c) $x \leq y \Rightarrow y_{a} \rightarrow z \leq x_{a} \rightarrow z$

(d) $(x \vee y)_{a} \rightarrow z=\left(x_{a} \rightarrow z\right) \wedge\left(y_{a} \rightarrow z\right)$.

Proof: Suppose $L$ be a skew Heyting algebra. Let $x, y, z \in$ $a \uparrow$. Clearly $a \uparrow$ is a skew Heyting algebra. If $x \leq y$, then $x$ $a \rightarrow y=(y \vee x \vee y)_{y} \rightarrow y=y_{y} \rightarrow y=1$. Thus (a) $\Rightarrow$ (b) holds. Using the fact that $z \uparrow$ is a Heyting algebra and $L$ is conormal, whenever $x \leq y$ we have

$\left(y_{a} \rightarrow z\right) \wedge\left(x_{a} \rightarrow z\right)$

$$
\begin{aligned}
& =\left((z \vee y \vee z)_{z} \rightarrow z\right) \wedge\left((z \vee x \vee z)_{z} \rightarrow z\right) \\
& =\{(z \vee y \vee z) \vee(z \vee x \vee z)\}_{z} \rightarrow z \\
& =\{(z \vee x \vee z) \vee(z \vee y \vee z)\}_{z} \rightarrow z \\
& =(z \vee x \vee y \vee z)_{z} \rightarrow z \\
& =(z \vee y \vee z)_{z} \rightarrow z \\
& =y_{a} \rightarrow \mathrm{z} .
\end{aligned}
$$

This shows that $(\mathrm{a}) \Rightarrow(\mathrm{c})$. From the fact that $z \uparrow$ is a Heyting algebra, we obtain the following result

$\left(x_{a} \rightarrow z\right) \wedge\left(y_{a} \rightarrow z\right)$

$$
\begin{aligned}
& =\left((z \vee x \vee z)_{z} \rightarrow z\right) \wedge\left((z \vee y \vee z)_{z} \rightarrow z\right) \\
& =\{(z \vee x \vee z) \vee(z \vee y \vee z)\}_{z} \rightarrow z \\
& =(z \vee x \vee y \vee z)_{z} \rightarrow z \\
& =(x \vee y)_{a} \rightarrow z
\end{aligned}
$$

Consequently (a) $\Rightarrow(d)$. To prove the converse, we show that for any $a \in L$, the semi-Heyting algebra $a \uparrow$ is a Heyting algebra. For this it suffices to show that each of the conditions (b), (c) and (d) implies that $(x \wedge y)_{a} \rightarrow x=1$. Suppose (b) holds. Let $a \in L$ such that $x, y \in a \uparrow$. Then $x \wedge y=y \wedge x$ and $(y \wedge x) \vee x=x \Rightarrow y \wedge x \leq x \Rightarrow x \wedge y \leq x$. From the given assumption we obtain that $(x \wedge y)_{a} \rightarrow x=1$. Now assume that (c) holds. Clearly $x, y \in a \uparrow$ implies that $x \wedge y \leq x$. Then by (c), we have $x_{a} \rightarrow z \leq(x \wedge y)_{a} \rightarrow z$ for any $z \in L$. Taking 
$z=x$ we get $x a_{a} \rightarrow x \leq(x \wedge y)_{a} \rightarrow x$. This inturn implies that $1 \leq(x \wedge y)_{a} \rightarrow x$. Hence $(x \wedge y)_{a} \rightarrow x=1$. Finally let (d) holds. Then for any $x, y \in a \uparrow, 1=x_{a} \rightarrow x=(x \vee(x \wedge y))_{a} \rightarrow x=\left(x_{a} \rightarrow\right.$ $x) \wedge\left((x \wedge y)_{a} \rightarrow x\right)=1 \wedge\left((x \wedge y)_{a} \rightarrow x\right)$. Therefore $(x \wedge y)_{a} \rightarrow x=$ 1.

In the following theorem we give another characterization of a skew semi-Heyting algebra.

Theorem 3.9: Let $L$ be a skew semi-Heyting algebra.Then $L$ is a skew Heyting algebra if and only if for all $b \in L$, the binary operation $b \rightarrow$ on $b \uparrow$ is the same as the induced binary operation $\rightarrow$ on $L$.

Proof: Suppose $L$ be a skew Heyting algebra. Thus for any $b \in L, b \uparrow$ is a Heyting algebra so that it is a skew Heyting algebra. Since the binary operation $\rightarrow$ on a skew Heyting algebra is unique, $x_{b} \rightarrow y=x \rightarrow y$ for any $x, y \in b \uparrow$ (see Lemma 2.9). Conversely suppose $x_{b} \rightarrow y=x \rightarrow y$ for any $x, y \in b \uparrow$. Since $b \uparrow$ is semi-Heyting algebra we need to prove that $(x \wedge y)_{b} \rightarrow x=1$. Using (SH4) for the semi-Heyting algebra $x \uparrow$ we get,$(x \wedge y)_{b} \rightarrow x=(x \wedge y) \rightarrow x=(x \vee(x \wedge y) \vee x)_{x} \rightarrow x=x$ $x \rightarrow x=1$. Hence $b \uparrow$ is a Heyting algebra and therefore $L$ is a skew Heyting algebra.

Consider the skew semi-Heyting algebra $L$ given by Example 3.3 whose induced binary operation $\rightarrow$ is defined by Table 1. In this example the induced binary operation $\rightarrow$ on $L$ is the same as the binary operation ${ }_{b} \rightarrow$ defined on $b \uparrow$ for each $b \in L$. Therefore this skew semi-Heyting algebra is a skew Heyting algebra.

In the next theorem we give an axiomatization for a skew semi-Heyting algebra.

Theorem 3.10: Let $(L, \vee, \wedge, \rightarrow, 0,1)$ be an algebra of type $(2,2,2,0,0)$ such that $(L, \vee, \wedge, 0,1)$ is a costrongly distributive skew lattice and let $b \in L$. Then $(L, \vee, \wedge, \rightarrow, 1)$ is a skew semiHeyting algebra if and only if the following conditions hold

(a) $x_{b} \rightarrow x=1$ for all $x \in b \uparrow$

(b) $x \wedge\left(x_{b} \rightarrow y\right)=x \wedge y$ for all $x, y \in b \uparrow$

(c) $x \wedge\left(y_{b} \rightarrow z\right)=x \wedge\left((x \wedge y)_{b} \rightarrow z\right)$ for all $x, y, z \in b \uparrow$

(d) $x \wedge\left(y_{b} \rightarrow z\right)=x \wedge\left(y_{b} \rightarrow(x \wedge z)\right)$ for all $x, y, z \in b \uparrow$

(e) $y \leq(x \rightarrow y)$ for all $x, y \in L$

(f) $x \rightarrow y=(y \vee x \vee y)_{y} \rightarrow y$ for all $x, y \in L$.

Proof: suppose $L$ be a skew semi-Heyting algebra. Then for any $b \in L, b \uparrow$ is a semi-Heyting algebra and hence by Theorem 2.4 (c) and (d) hold. But (a) and (b) hold directly from the definition of semi-Heyting algebra. Condition (e) and (f) is direct from the assumption. Since $L$ is co-strongly distributive skew lattice, for any $b \in L, b \uparrow$ is a lattice. So to prove the converse it is enough to prove that $b \uparrow$ is a semi-Heyting algebra and for this we show that $x \wedge\left(y_{b} \rightarrow\right.$ $z)=x \wedge\left((x \wedge y)_{b} \rightarrow(x \wedge z)\right)$. Then from (c) we have $x \wedge\left(y_{b} \rightarrow\right.$ $z)=x \wedge\left((x \wedge y)_{b} \rightarrow z\right)$, and by $(\mathrm{d})$ we get $x \wedge\left((x \wedge y)_{b} \rightarrow z\right)=x \wedge$ $\left((x \wedge y)_{b} \rightarrow(x \wedge z)\right.$. Hence $x \wedge\left(y_{b} \rightarrow z\right)=x \wedge\left((x \wedge y)_{b} \rightarrow(x \wedge z)\right)$. Now for $b \in L$ and $x, y, z \in b \uparrow$, set $x_{b} \rightarrow y=x \rightarrow y$. Clearly (e) implies that $x \rightarrow y \in y \uparrow \subseteq b \uparrow$. Thus the restriction ${ }_{b} \rightarrow$ of $\rightarrow$ to $b \uparrow$ is well defined(see [6]). Since $b \uparrow$ is commutative (a), (b) and, (c) and (d) for $\rightarrow$ simplify respectively to (SH4), (SH2) and (SH3) for ${ }_{b} \rightarrow$ making $b \rightarrow$ is the binary operation on $b \uparrow$. This shows that for each $b \in L,(b \uparrow, \vee, \wedge, b \rightarrow, b, 1)$ is a semiHeyting algebra. Therefore using (f) it is possible to define an induced binary operation $\rightarrow$ on $L$ by $x \rightarrow y=(y \vee x \vee y)_{y} \rightarrow y$ that makes $L$ is a skew semi-Heyting algebra.

Corollary 3.11: Let $(L, \vee, \wedge, \rightarrow, 0,1)$ be an algebra of type $(2,2,2,0,0)$ such that $(L, \vee, \wedge, 0,1)$ is a costrongly distributive skew lattice and let $b \in L$. Then $(L, \vee, \wedge, \rightarrow, 1)$ is a skew semiHeyting algebra if and only if the following conditions hold

(a) $x \leq\left(x_{b} \rightarrow y\right)_{b} \rightarrow y$

(b) $x \wedge\left(x_{b} \rightarrow y\right)=x \wedge y$ for all $x, y \in b \uparrow$

(c) $x \wedge\left(y_{b} \rightarrow z\right)=x \wedge\left((x \wedge y)_{b} \rightarrow z\right)$ for all $x, y, z \in b \uparrow$

(d) $x \wedge\left(y_{b} \rightarrow z\right)=x \wedge\left(y_{b} \rightarrow(x \wedge z)\right)$ for all $x, y, z \in b \uparrow$

(e) $y \leq(x \rightarrow y)$ for all $x, y \in L$

(f) $x \rightarrow y=(y \vee x \vee y)_{y} \rightarrow y$ for all $x, y \in L$.

Proof: From (b) we have $1 \wedge\left(1_{b} \rightarrow x\right)=1 \wedge x=x \Rightarrow 1$ ${ }_{b} \rightarrow x=x$. Thus from (a) we get $1 \leq\left(1_{b} \rightarrow x\right)_{b} \rightarrow x$, which implies that $1 \leq x_{b} \rightarrow x$. Thus $x_{b} \rightarrow x=1$. Therefore $b \uparrow$ is a semi-Heyting algebra. The rest of the proof follows from the above theorem.

Theorem 3.12: Let $(L, \vee, \wedge, \rightarrow, 1)$ be a skew semi-Heyting algebra. Then for any $x, y \in L$, the algebra $([x, y], \vee, \wedge, x \rightarrow, y)$ is a skew semi-Heyting algebra.

Proof: Suppose $L$ be a skew semi-Heyting algebra and $x, y \in L$. We show that $[a, y]$ is a semi-Heyting algebra for any $a \in[x, y]$. Clearly $[x, y]$ is a co-strongly distributive skew lattice. Since $L$ is a skew semi-Heyting algebra, for any $x \in L, x \uparrow$ is a semi-Heyting algebra and therefore $[x, y]$ is a semi-Heyting algebra(see [7]). Again by the same reason $[a, y]$ is a semiHeyting algebra. Finally using (Theorem 3.9) one can define $x \rightarrow$ on $[x, y]$ by $c_{x} \rightarrow d=(d \vee c \vee d)_{d} \rightarrow d$ so that $[x, y]$ is a skew semi-Heyting algebra.

Lemma 3.13: Let the algebra $([x, y], \vee, \wedge, x \rightarrow, y)$ be a skew semi-Heyting algebra. Then the algebra $([x, y], \vee, \wedge, x \rightarrow, x, y)$ is a Heyting algebra.

Proof: Let $a, b \in[x, y]$. Then $(a \wedge b)_{x} \rightarrow a=(a \vee(a \wedge b) \vee$ $a)_{a} \rightarrow a=a \quad{ }_{a} \rightarrow a=y$. Thus $([x, y], \vee, \wedge, x \rightarrow, x, y)$ becomes a Heyting algebra.

Theorem 3.14: Let $L$ be a binormal skew semi-Heyting algebra and $b \in L$. Let $\theta$ be the relation on $b \uparrow$ defined by $(x, y) \in \theta$ if and only if $b_{0} \wedge x=b_{0} \wedge y$ for some $b_{0} \in b \uparrow$.

Then $\theta$ is a congruence relation on $b \uparrow$.

Proof: Suppose $L$ be a binormal skew semi-Heyting algebra. Clearly $\theta$ is reflexive and symmetric. Let $b \in$ $L$ and $, x, y, z \in b \uparrow$ such that $(x, y) \in \theta$ and $(y, z) \in \theta$. This indicates that there exist $b_{1}$ and $b_{2} \in b \uparrow$ such that $b_{1} \wedge x=b_{1} \wedge y$ and $b_{2} \wedge y=b_{2} \wedge z$. Then $b_{2} \wedge b_{1} \wedge x=b_{2} \wedge b_{1} \wedge y=b_{1} \wedge$ $b_{2} \wedge y=b_{1} \wedge b_{2} \wedge z$. This implies that $(x, z) \in \theta$ and hence $\theta$ is transitive. Therefore $\theta$ is an equivalence relation. Let $\left(x_{1}, y_{1}\right) \in \theta$ and $\left(x_{2}, y_{2}\right) \in \theta$. Then there exist $b_{1}, b_{2} \in b \uparrow$ such that $b_{1} \wedge x_{1}=b_{1} \wedge y_{1}$ and $b_{2} \wedge x_{2}=b_{2} \wedge y_{2}$. Now we show that $\left(x_{1} \wedge x_{2}, y_{1} \wedge y_{2}\right) \in \theta$, and $\left(x_{1} \vee x_{2}, y_{1} \vee y_{2}\right) \in \theta$. Thus $b_{1} \wedge x_{1} \wedge b_{2} \wedge x_{2}=b_{1} \wedge b_{2} \wedge x_{1} \wedge x_{2}$ so that $b_{1} \wedge y_{1} \wedge b_{2} \wedge y_{2}=$ $b_{1} \wedge b_{2} \wedge y_{1} \wedge y_{2}$. Then $b_{1} \wedge b_{2} \wedge x_{1} \wedge x_{2}=b_{1} \wedge x_{1} \wedge b_{2} \wedge x_{2} \wedge$ $b_{1} \wedge y_{1} \wedge b_{2} \wedge y_{2}=b_{1} \wedge b_{2} \wedge y_{1} \wedge y_{2}$. Hence $\left(x_{1} \wedge x_{2}, y_{1} \wedge y_{2}\right) \in \theta$. Similarly, since $b \uparrow$ is distributive lattice we have

$$
\begin{aligned}
b_{1} \wedge b_{2} \wedge\left(x_{1} \vee x_{2}\right) & =\left(b_{1} \wedge b_{2} \wedge x_{1}\right) \vee\left(b_{1} \wedge b_{2} \wedge x_{2}\right) \\
& =\left(b_{2} \wedge b_{1} \wedge x_{1}\right) \vee\left(b_{1} \wedge b_{2} \wedge x_{2}\right) \\
& =\left(b_{2} \wedge b_{1} \wedge y_{1}\right) \vee\left(b_{1} \wedge b_{2} \wedge y_{2}\right)
\end{aligned}
$$




$$
=b_{1} \wedge b_{2} \wedge\left(y_{1} \vee y_{2}\right) .
$$

which shows that $\left(x_{1} \vee x_{2}, y_{1} \vee y_{2}\right) \in \theta$. Finally we obtain $b_{1} \wedge b_{2} \wedge\left(x_{1} \rightarrow x_{2}\right)$

$$
\begin{aligned}
& =b_{1} \wedge b_{2} \wedge\left(\left(b_{1} \wedge b_{2} \wedge x_{1}\right) \rightarrow\left(b_{1} \wedge b_{2} \wedge x_{2}\right)\right) \\
& =b_{1} \wedge b_{2} \wedge\left(\left(b_{2} \wedge b_{1} \wedge x_{1}\right) \rightarrow\left(b_{1} \wedge b_{2} \wedge x_{2}\right)\right) \\
& =b_{1} \wedge b_{2} \wedge\left(\left(b_{2} \wedge b_{1} \wedge y_{1}\right) \rightarrow\left(b_{1} \wedge b_{2} \wedge y_{2}\right)\right) \\
& =b_{1} \wedge b_{2} \wedge\left(\left(b_{1} \wedge b_{2} \wedge y_{1}\right) \rightarrow\left(b_{1} \wedge b_{2} \wedge y_{2}\right)\right) \\
& =b_{1} \wedge b_{2} \wedge\left(y_{1} \rightarrow y_{2}\right) .
\end{aligned}
$$

Since $b \uparrow$ is a lattice $b_{1} \wedge b_{2} \in b \uparrow$, thus $\left(x_{1} \rightarrow x_{2}, y_{1} \rightarrow y_{2}\right) \in \theta$. Therefore $\theta$ is a congruence relation on $b \uparrow$.

\section{REFERENCES}

[1] J. Leech, "Skew lattices in rings," Algebra Universalis, vol. 26, no. 1, pp. 48-72, 1989.

[2] — , "Skew boolean algebras," Algebra Universalis, vol. 27, no. 4, pp. 497-506, 1990.

[3] —_, "Normal skew lattices," in Semigroup Forum, vol. 44, no. 1, 1992, pp. $1-8$.

[4] T. Skolem, "Logico-combinatorial investigations in the satisfiability or provability of mathematical propositions: a simplified proof of a theorem by 1. löwenheim and generalizations of the theorem," From Frege to Gödel. A Source Book in Mathematical Logic, vol. 1931, pp. 252-263, 1879 .

[5] G. Birkhoff, Lattice theory. American Mathematical Soc., 1940, vol. 25.

[6] K. Cvetko-Vah, "On skew heyting algebras," arXiv preprint arXiv:1410.8780, 2014.

[7] H. Sankappanavar, "Semi-heyting algebras: An abstraction from heyting algebras," in Actas del IX Congreso Dr. A. Monteiro, 2007, pp. 33-66.

[8] G. Rao, B. Assaye, and R. Prasad, "L-almost distributive lattices," AsianEuropean Journal of Mathematics, vol. 4, no. 01, pp. 171-178, 2011. 